Gynäkologisch--
geburtshilfliche
Rundschau

\section{U. Haller, Zürich}

Gynäkol Geburtshilfliche Rundsch 1999;39:109

\section{Editorial}

Die Herausgeber freuen sich, dass die Schweizerische Gesellschaft für Gynäkologie und Geburtshilfe (SGGG) die Abstracts für ihre Jahrestagung vom 24.-26. Juni 1999 in Davos in der Rundschau publiziert. Damit geht das alte Postulat in Erfüllung, für unsere jungen wissenschaftlich tätigen Kolleginnen und Kollegen zitierfähige Abstracts zur Verfügung zu stellen. Ursprünglich war vorgesehen, einen Abstractband zusammen mit der gemeinsamen Tagung der Bayerischen und Österreichischen Gesellschaft für Gynäkologie und Geburtshilfe, welche im gleichen Monat in München stattfindet, herauszugeben. Dies war aus zeitlichen Gründen leider nicht mehr möglich, so dass sich die Herausgeber einigten, das vorliegende Heft Nr. 3 zur Hälfte mit dem üblichen Inhalt und zur anderen Hälfte mit den Abstracts für die Schweizerische Jahrestagung zu gestalten.

Für unseren akademischen Nachwuchs wurden während der letzten Jahre in unseren drei deutschsprachigen Ländern die Ansprüche zur Erfüllung der Publikationsleistung hochgetrieben. So wurden denn auch Kriterien wie der Impact Factor unverhältnismässig in ihrer Bedeutung gestärkt. Unseren jungen, in der Grundlagen- und in der klinischen Forschung tätigen Kolleginnen und Kollegen soll deshalb die Möglichkeit gegeben werden, ihre mehrheitlich ausgezeichneten Referateabstracts und Posterbeiträge, die mit viel Mühe und grossem Engagement erarbeitet wurden, zitierfähig zu machen.

Verlag und Herausgeber haben während der letzten Monate zusammen mit dem Beirat und mit engagierten Mitgliedern, insbesondere der österreichischen Gesellschaft, an einem neuen modernen Konzept für unsere Zeitschrift gearbeitet, welches vermehrt auch den Anliegen unserer Gynäkologinnen und Gynäkologen in der Praxis Rechnung trägt. Dies machte auch strukturelle und organisatorische Veränderungen notwendig, über die wir in einer der nächsten Nummern berichten werden. Für die Schweizerische Gesellschaft stellt sich in diesem Zusammenhang einmal mehr die Frage, ob für die Rundschau eine Rückkehr als Organ der Fachgesellschaft wieder im Bereich der Möglichkeit liegt. Es sei schon jetzt allen aktiven Mitgliedern, die sich mit Tatkraft und Ideen für dieses neue Konzept unserer Zeitschrift eingesetzt haben, gedankt. 